\title{
The Flawed Scientific Basis of the Altered Nuclear Transfer-Oocyte Assisted Reprogramming (ANT-OAR) Proposal
}

\author{
W. Malcolm Byrnes
}

Published online: 22 May 2007

(C) Humana Press Inc. 2007

\begin{abstract}
First put forth in June 2005, the altered nuclear transfer-oocyte assisted reprogramming (ANT-OAR) proposal has been promoted as an ethically-acceptable alternative to the embryo-destructive methods now used to obtain embryonic stem cells. According to its proponents, the goal of ANT-OAR is to use the cloning process to create a pluripotent stem cell. This would be achieved through overexpression of the transcription factor Nanog (or a hypothetical substitute) both in the enucleated egg cell and in the somatic cell prior to transfer of its nucleus. Although the ethical acceptability of ANT-OAR has been publicly debated, its scientific feasibility has not. This paper aims to help rectify this situation. It argues that ANT-OAR, as currently conceived, cannot realistically work. It presents evidence from the scientific literature showing that Nanog cannot single-handedly establish pluripotency in cells, but rather works together with a network of other transcription factors to maintain pluripotency. It argues that ANT-OAR is based on a flawed understanding of stem cell biology, and emphasizes that, in this debate about embryonic stem cells, scientists must strive to accurately and realistically assess the feasibility of the embryo research strategies they propose.
\end{abstract}

Keywords Altered nuclear transfer-ANT .

Altered nuclear transfer-oocyte assisted reprogramming . ANT-OAR · Embryonic stem cells $\cdot$ Bioethics $\cdot$ Nanog

W. M. Byrnes $(\square)$

Department of Biochemistry and Molecular Biology, College of Medicine, Howard University, 520 W Street, NW, Washington, DC 20059, USA

e-mail: wbyrnes@howard.edu

\section{What is ANT-OAR?}

In June 2005, a proposal known as altered nuclear transferoocyte assisted reprogramming (ANT-OAR) was put forth as an ethically-acceptable alternative to the currently available method of obtaining embryonic stem cells, which involves their extraction from human IVF embryos using a procedure that destroys the embryos [1]. The ANT-OAR proposal is a variant of another proposal, known as altered nuclear transfer (ANT; now known as ANT-Cdx2) that was unveiled at the December 2004 meeting of the U.S. President's Council on Bioethics by William Hurlbut [2]. ANT-Cdx2 involves altering a somatic cell nucleus prior to transfer so that it contains a transgene for a short RNA molecule that can target and destroy the Cdx2 mRNA transcript (through RNA interference; see [3]). After transfer, expression from the transgene effectively removes $\mathrm{Cd} 2 \mathrm{2}$ from the developing embryo. $\mathrm{Cdx} 2$ is a transcription factor essential for formation of the trophectoderm [4], and embryos that lack $\mathrm{Cdx} 2$ become structurally disorganized and cannot implant. However, since $\mathrm{Cdx} 2$ is not expressed until the morula stage ([5]; See Appendix Box 1), its knockdown in an ANT-Cdx2 embryo does not take effect until that stage. As a result, the Cdx2-deficient embryo up until the morula stage would be essentially normal. This problem - the fact that ANT-Cdx2 produces an embryo that is normal during at least part of its development-led some to question the ethical acceptability of ANT-Cdx2. As a result of this problem, ANT-OAR was proposed.

With ANT-OAR, the goal was to have the biochemical or genetic alteration take effect immediately in the newlycloned embryo, not after a delay as in ANT-Cdx2. The idea was to over-express the transcription factor Nanog both in the somatic cell whose nucleus would be transferred during the somatic cell nuclear transfer (SCNT) step and in the 
enucleated oocyte that would accept the altered somatic nucleus [1]. Nanog had been shown to play a central role in maintaining pluripotency in mouse embryonic stem cells $[6$, 7]. The hope was that the expression of NANOG in the somatic cell prior to the removal and transfer of its nucleus, together with the expression of Nanog from mRNA injected into the oocyte itself, would produce a one-celled entity that was, from its first moment of existence, a pluripotent stem cell. In this way, and unlike the situation with ANT-Cdx2, there would be no gap in time during which the cloned entity would be normal. Nanog would directly convert the entity into a pluripotent stem cell, bypassing the embryo stage altogether.

Despite having been designed to solve the ethical problems with ANT-Cdx2, ANT-OAR was not met with an unqualified endorsement. Soon after its announcement, some philosophers presented strong arguments that it was not ethically sound [8]. However, from the time of its inception until now, the scientific feasibility of ANT-OAR has never been publicly debated to any great extent. ${ }^{1}$ Part of the reason for this neglect is that the scientists supporting ANT-OAR have not provided much detail regarding how ANT-OAR is supposed to work. Recently, though, one of the original signatories of the ANT-OAR proposal, biologist Nicanor Austriaco, presented a detailed justification of the scientific feasibility of ANT-OAR [9]. This justification by Austriaco now provides the starting point for a scientific debate.

\section{ANT-OAR is Based on Flawed Science}

To support his arguments for the scientific feasibility of ANT-OAR, Austriaco cites a number of scientific papers relating to Nanog and its role in cellular pluripotency. However, a careful reading of these papers, and others, shows that Nanog, powerful though it is, is not able to single-handedly transform a newly-cloned embryo into a pluripotent stem cell. These papers, discussed below, reveal that Nanog does not act alone in maintaining pluripotency, but rather is part of a complex network of transcription factors that operate within a particular context in embryonic stem cells.

Before embarking on a discussion of Nanog and its interdependent role in pluripotency, it is important to emphasize that the processes that maintain pluripotency and self-renewal in embryonic stem cells are complex and involve a number of parts. They involve not only the core

\footnotetext{
${ }^{1}$ It should be noted that scientific aspects of ANT-OAR were considered at the conference Stem Cells: What Future for Therapy? - Scientific Aspects and Bioethical Problems held at the Vatican, Rome, on June 14-16, 2006.
}

set of regulatory transcription factors Oct4, Sox 2 and Nanog that will be discussed here; these activate or repress several hundred or more downstream target genes that control the decision between self-renewal and differentiation. They also involve a set of extracellularly-stimulated signaling pathways whose role is to activate this regulatory core of factors. Thus, Nanog, Oct4 and Sox 2 are central players positioned between extracellular signals and downstream target genes. In considering the signaling pathways of self-renewal and pluripotency, it is also important to bear in mind that these pathways are not all the same among species. The particular set of pathways involved in maintaining pluripotency in mouse embryonic stem cells, for example, overlaps with, but is not identical to, the set of pathways important in human embryonic stem cells. An example is the LIF/STAT (leukemia inhibitory factor/ signal transducer and activator of transcription) pathway, which is important in mouse, but not human, embryonic stem cells. Finally, in addition to signaling pathways, epigenetic effects such as the modification of chromatin structure and dynamics are involved in regulating the expression of key lineage-specific genes. Several recent reviews present overviews of the various aspects of pluripotency and selfrenewal in embryonic stem cells [10-13].

Turning back to Nanog and its role, as mentioned above, a number of recent studies have shown that Nanog, though important, is only one part of a network of transcription factors that maintains pluripotency. First, Loh et al. [14] demonstrated that Nanog, Oct4 and Sox 2 work together to "control a cascade of pathways that are intricately connected to govern pluripotency, self-renewal, genome surveillance and cell fate determination" in mouse embryonic stem cells. Moreover, they found that Nanog and Oct4 bind to many of the same downstream target genes and, indeed, co-localize to the same regions of these genes' promoters. In related work, Boyer et al. [15] found that, in human embryonic stem cells, NANOG together with SOX2 and OCT4 "collaborate to form regulatory circuitry consisting of autoregulatory and feedforward loops" that affect the expression of hundreds of downsteam genes. These authors also found that the levels and functions of NANOG, SOX2 and OCT4 were "tightly linked," and that the relative stoichiometry of the three factors was critical for keeping the regulatory circuitry intact.

Second, a paper by Rodda et al. [16] shows that Oct4 and Sox 2 physically interact with each other at the nanog gene promoter to regulate Nanog expression. These authors conclude that Oct4-Sox2 complex is "at the top of the pluripotent regulatory network hierarchy." Furthermore, an elegant paper by a Chinese group (Pan et al. [17]) confirms that Oct4 regulates Nanog expression at the transcriptional level, and makes the important conclusion that "Oct4 and Nanog need to function in parallel in maintaining [embryonic 
stem] cell self-renewal and that neither is dispensable nor capable of compensating [for] the role of the other." Another conclusion they reach is that a "FoxD3-Nanog-Oct4 loop anchors an interdependent network of transcription factors that regulate stem cell pluripotency." (FoxD3, a member of the forkhead family, is a repressive transcription factor that is required for pluripotency in the mouse.) Thus, with these studies, we see that Nanog, though important, is only one part of a complex network of transcription factors that work together to establish pluripotency.

Third, Austriaco states that a paper by Silva et al. [18] presents evidence that "overexpression of Nanog alone leads to the reprogramming of neural or skin cells into cells that show embryonic stem cell characteristics." However, this statement is misleading. The paper does show that the fusion of neural stem cells containing elevated levels of Nanog with embryonic stem cells allows the neural stem cell epigenome to be "reset completely to a state of pluripotency." Nanog thus plays a "dominant role in instating pluripotency in an [embryonic stem] cell hybrid." But two caveats are important here. First, Nanog works in this case in an embryonic stem cell hybrid, not a newlycloned embryo, as would be the case for ANT-OAR. Second, as Silva and colleagues emphasize, "Nanog does not operate alone to transfer pluripotency, but acts in conjunction with additional [embryonic stem] cell machinery" [9]. This particular set of machinery, which is particular to embryonic stem cells, would not be expected to be present just after the SCNT event in the new embryo. It is misleading, then, to state as Austriaco does that overexpression of Nanog alone leads to pluripotency.

Fourth, while the paper by Silva and coworkers, and one by Ivanova et al. [19] highlight the important role of Nanog in promoting and maintaining stem cell pluripotency, another recent paper shows that Nanog is not needed for the reprogramming of an adult cell to pluripotency. Takahashi and Yamanaka [20] demonstrate that overexpression of a set of only four factors-Oct4, Sox2, c-Myc and Klf4 - can induce pluripotency in adult mouse fibroblast cells. And, although the pluripotent cells that result do not perfectly match embryonic stem cells, they have many of the characteristics of embryonic stem cells. Moreover, when microinjected into blastocysts, they contribute to all three germ layers of 13.5-day post implantation embryos into which the blastocysts can develop (although they apparently do not support development beyond 16 days). These results nevertheless provide hope that one of the holy grails of stem cell biology [21] - the reprogramming of an adult cell directly to a pluripotent cell so that embryos are not used at all — may be achievable. It is also interesting that Nanog was absent from the list of four factors; this indicates that overexpression of Nanog is not necessary for the conversion of an adult cell to a pluripotent cell. Thus, while Nanog is required to maintain pluripotency in embryonic stem cells, it apparently is not required to establish pluripotency in adult cells. Given these facts, one is compelled to ask: How likely would it be that Nanog could single-handedly establish pluripotency in a newly-cloned embryo?

These studies show that Nanog does not act alone, but it is part of a complex network of transcription factors that together maintain pluripotency in embryonic stem cells. Other studies emphasize that events occurring in the developing embryo prior to the onset of Nanog expression help prepare the embryo for the proper spatial and temporal expression of Nanog. For example, Hamatani et al. [22] show that preimplantation gene activation occurs in a series of waves in the embryo, with the formation of each wave dependent upon the one before. The nanog gene is "turned on" around the 8-cell stage during a phenomenon called "mid-preimplantation gene activation." How might the misexpression of Nanog out of sequence and out of context affect its ability to fulfill its normal role as a master regulator of pluripotency?

\section{A Speculative Proposal}

Altogether, the scientific evidence presented above suggests that supporters of ANT-OAR are in error when they attribute almost unlimited power to Nanog as a pluripotency-inducing factor. They misunderstand the scientific facts and, as a result, are unable to realistically assess the feasibility of the ANT-OAR proposal.

The disjunction between the scientific facts and the hopeful fiction entertained by the supporters of ANT-OAR highlights a serious problem: the proposal is entirely speculative. Furthermore, since it is not anchored in scientific reality, it can become whatever its supporters wish it to be. The fact that Nanog cannot work to reprogram the newly-cloned embryo in the manner predicted apparently does not pose a problem for ANT-OAR proponents. In this case, a different factor or set of factors simply can be found to fill Nanog's perceived role. It is almost as if the idea of ANT-OAR is so urgently desired that it is irrelevant whether or not it can work biologically.

A related question is this: if a cocktail of factors is found to be required to reprogram the cloned cell into a pluripotent cell, then why would one want to go through the cloning step at all? Why not just try to reprogram an adult cell directly into a pluripotent cell? Going this other route would remove the ethical problems associated with the initiation of a human life, however transiently it might exist, through the cloning event. Importantly, as the work by Takahashi and Yamanaka shows [20], the reprogramming of an adult cell might indeed be possible. 
One can get a clearer sense of some of the problems with ANT-OAR by examining Austriaco's statement summarizing the differences he sees between ANT (it is not clear whether he is writing about ANT-Cdx2, ANT-OAR, or both; apparently he conflates the two even though they are different) and SCNT:

[T] here is no time when the ANT and SCNT products are identical, because the reprogramming that goes on in the two cells involves two different processes, which begin with the same nucleus but follow different, non-overlapping trajectories and pass through different epigenetic states... With ANT, the reprogramming of the somatic cell nucleus begins prior to its transfer into the enucleated oocyte. In contrast, with SCNT, the reprogramming of the nucleus begins after its transfer [emphasis original]. Therefore, at fusion with the enucleated cytoplasm, the ANT nucleus is already distinguishable from the SCNT nucleus. They are different nuclei with different epigenetic states [9].

From these statements a question arises: Why would one expect the reprogramming process that might take place in an ANT-OAR-derived embryo to be different from the one that takes place in the SCNT-derived embryo? This has not been tested experimentally (See Appendix Box 2). No one knows what might happen when Nanog is overexpressed in an enucleated mouse egg prior to transfer. No one knows how similar or different the "epigenetic states" of the nuclei of the two cloned entities will be. Will factors in the oocyte cytoplasm overwhelm the presence of Nanog and steer development on a normal course? Or, will Nanog prove fatal in the one-celled cloned embryo, where the milieu is quite different from the milieu Nanog normally encounters in the morula? Or, will Nanog transform the embryo into a pluripotent stem cell, as ANT-OAR proponents hope it will. Given all of the scientific evidence presented above, this third possibility seems unlikely. And yet, for ANT-OAR proponents, this is what will happen.

\section{Conclusion}

ANT-OAR proposes that the overexpression of the transcription factor Nanog, or a hypothetical substitute, will immediately transform the cloned human entity in which it is expressed into a pluripotent stem cell, thereby bypassing the embryo stage altogether. In this way, it claims to be an ethical alternative to the currently available, embryodestructive method of obtaining stem cells. However, the scientific evidence is overwhelming that Nanog cannot single-handedly establish pluripotency in stem cells, and its ability to do so in the foreign milieu of a newly cloned embryo would be even less likely. Moreover, while it remains a possibility that a different transcription factor (other than Nanog) might be found in the future that will act to single-handedly establish pluripotency in a cloned embryo, based on the evidence with Nanog, which highlights the complex and interdependent nature of the pluripotent state, this seems unlikely. Finally, the example presented here of the flawed scientific basis of ANT-OAR emphasizes that it is important for scientists to realistically and honestly assess - on an ongoing basis - the feasibility of the stem cell research strategies they propose. Doing so, by participants on all sides of this politically-charged debate, would go a long way toward gaining the trust of a public increasingly skeptical about biomedical science.

Acknowledgements The author would like to thank Dr. Davor Solter of the Max-Planck Institute of Immunobiology, Freiburg, Germany, and Dr. Magdalena Zernicka-Goetz of the Gurdon Institute, University of Cambridge, U.K., for their review of earlier drafts of this paper.

\section{Appendix}

\section{Box 1 Controversy over the Timing of $\mathrm{Cdx} 2$ Expression}

Recently, there has been some controversy regarding whether or not $\mathrm{Cdx} 2$ might be present much earlier than the morula stage. A report by Deb et al. [23] seemed to show that Cdx2 mRNA is present in the zygote and, indeed, even in the oocyte, where its asymmetrical distribution determines, after fertilization, which cell of the two-celled embryo becomes trophectoderm (the late-dividing cell does), and which becomes the inner cell mass (the earlydividing cell) of the blastocyst. These results contradicted the two accepted models for pre-implantation development derived from lineage tracing experiments [24]. The first model predicts that one blastomere of the zygote has a bias toward becoming what is known as the embryonic part of the blastocyst, which contains the inner cell mass (ICM) and polar trophectoderm, and the other blastomere has a bias toward forming the abembryonic part, which contains the mural trophectoderm and more superficial ICM. Both blastomeres contribute to both parts; thus, blastomere fates are not strictly determined. Which blastomere contributes more to which part depends on subsequent cleavage patterns [25]. The second model predicts that the contributions of the two-cell blastomeres to the parts of the future blastocyst are completely random [26]. The Deb et al. paper contradicts both of these models because it predicts that the lineage fates of the blastomeres are already determinednot biased or random - at the two-cell stage. Notably, however, it has since come to light that the results of the 
Deb et al. paper may not be reliable. The editor of Science, in which the work was published, recently issued an "editorial expression of concern" [27]. The most recent news is that the paper likely will be retracted [28]. Nicanor Austriaco, in his recent paper on ANT-OAR ([9]; see main text), cites the Deb et al. paper in order to show that $\mathrm{Cdx} 2$ is present from the one-celled stage onward and therefore is not first expressed at the morula stage. Thus, in this scenario, a lack of $\mathrm{Cdx} 2$ in the ANT-Cdx2 entity would be "felt" from the very beginning. This would resolve the ethical problem that some had with ANT-Cdx2 and that led to the proposal of ANT-OAR, namely, the delay in the expression of $\mathrm{Cdx} 2$ (see main text). Nevertheless, the results of the Deb et al. paper also would have presented a conundrum, for how could the absence of a factor that is important so early on not have a profound effect on development? In such a situation, how could the embryo develop to the blastocyst stage at all? How could viable stem cells, which are extracted from the inner cell mass, have been obtained by Meissner and Jaenisch [3] in their study? The likelihood that the Deb et al. results are not reliable clears up this apparent conundrum; $\mathrm{Cdx} 2$ is, after all, expressed first in the morula, not the zygote.

\section{Box 2 Ethical Problems Associated with ANT-Cdx2 and ANT-OAR}

An ethical issue related to the experimental testing of both ANT-OAR and ANT-Cdx2 is this: even if they are found to work in mice - an extremely unlikely proposition for ANTOAR, although ANT-Cdx2 apparently does work in mice [3] - this does not mean that they will work in humans. Recently, it has become evident that the transcriptional network that regulates pluripotency in human embryonic stem cells is not exactly the same as the network that regulates pluripotency in mouse embryonic stem cells [29]. Moreover, there are differences between species with regard to some of the signaling pathways that regulate pluripotency and self-renewal. This suggests that what works in mice might not work in humans. To find out if the procedures work in humans, they will have to be tested using human embryos. (Note that any conceivable form of ANT has this difficulty). This is not a trivial issue, as Davor Solter has pointed out [30]. The procedures will have to be experimentally verified in the laboratory, which involves a trial-and-error approach. How could this be done ethically on human embryos? During the verification process, and even after successful verification, there inevitably will be some individual "failures" in that some normal human embryos will be produced. What will be done with these embryos? Though apparently normal, they nevertheless might harbor defects that will go undetected. What woman would knowingly allow such embryos to be implanted and gestated in her uterus? (Moreover, would this not be an example of reproductive cloning, which most agree is ethically unwise?) How will these embryos be saved? Clearly, the ANT solution is not an ethically acceptable solution at all.

\section{References}

1. Arkes, H., Austriaco, N., Berg, T., Brugger, E. C., Cameron, N. M., Capizzi, J., et al. (2005). Production of pluripotent stem cells by oocyte assisted reprogramming: Joint statement with signatories. National Catholic Bioethics Quarterly, 5, 579-583. Also available at: www.eppc.org/publications/pubID.2374/pub_details.asp. Last accessed 1-02-07.

2. Hurlbut, W. (2004). Session 6: Seeking morally unproblematic sources of human stem cells. Transcript, President's Council on Bioethics, December 4, 2004. Available at: www.bioethics.gov/ transcripts/dec04/session6.html. Last accessed 9-17-06.

3. Meissner, A., \& Jaenisch, R. (2006). Generation of nuclear transfer-derived pluripotent ES cells from cloned Cdx2-deficient blastocysts. Nature, 439, 21221-21225.

4. Chawengsaksophak, K., de Graaf, W., Rossant, J., Deschamps, J., $\&$ Beck, F. (2004). Cdx2 is essential for axial elongation in mouse development. Proceedings of the National Academy of Sciences, 101, 7641-7645.

5. Strumpf, D., Mao, C.-A., Yamanaka, Y., Ralston, A., Chawengsaksophak, K., Beck, F., et al. (2005). Cdx2 is required for correct cell fate specification and differentiation of trophectoderm in the mouse blastocyst. Development, 32, 2093-2102.

6. Mitsui, K., Tokuzawa, Y., Itoh, H., Segawa, K., Murakami, M., Takahashi, K., et al. (2003). The homeoprotein Nanog is required for maintenance of pluripotency in mouse epiblast and ES cells. Cell, 113, 631-642.

7. Chambers, I., Colby, D., Robertson, M., Nichols, J., Lee, S., Tweedie, S., et al. (2003). Functional expression cloning of Nanog, a pluripotency sustaining factor in embryonic stem cells. Cell, 113, 643-656.

8. See articles on the Communio website at: www.communio-icr.com/ ant.htm, especially: Walker, A. Reasonable doubts. A reply to E. Christian Brugger. Communio 2005; 32:770-783. Last accessed 1-02-07.

9. Austriaco, N. (2006). The moral case for ANT-derived pluripotent stem cell lines. National Catholic Bioethics Quarterly, 6, 517-537.

10. Sun, Y., Li, H., Yang, H., Rao, M., \& Zhan, M. (2006). Mechanisms controlling embryonic stem cell self-renewal and differentiation. Critical Reviews in Eukaryotic Gene Expression, 16, 211-231.

11. Noggle, S., James, D., \& Brivanlou, A. (2005). A molecular basis for human embryonic stem cell pluripotency. Stem Cell Rev, 1, $111-118$.

12. Boyer, L., Mathur, D., \& Jaenisch, R. (2006). Molecular control of pluripotency. Current Opinion in Genetics \& Development, 16, 455-462.

13. Gan, Q., Yoshida, T., McDonald, O., \& Ownes, G. (2007). Concise Review: Epigenetic mechanisms contribute to pluripotency and cell lineage determination of embryonic stem cells. Stem Cells, 25, 2-9.

14. Loh, Y.-H., Wu, Q., Chew, J.-L., Vega, V. B., Zhang, W., Chen, X., et al. (2006). The Oct4 and Nanog transcription network regulates pluripotency in mouse embryonic stem cells. Nature Genetics, 38, 431-440. 
15. Boyer, L., Lee, T., Cole, M., Johnstone, S. E., Levine, S. S., Zucker, J. P., et al. (2005). Core transcriptional regulatory circuitry in human embryonic stem cells. Cell, 122, 947-956.

16. Rodda, D., Chew, J.-L., Lim, L.-H., Loh, Y. H., Wang, B., $\mathrm{Ng}, \mathrm{H} . \mathrm{H}$., et al. (2005). Transcriptional regulation of Nanog by OCT4 and SOX2. Journal of Biological Chemistry, 280, 24731-24737.

17. Pan, G., Li, J., Zhou, Y., Zheng, H., \& Pei, D. (2006). A negative feedback loop of transcription factors that controls stem cell pluripotency and self-renewal. FASEB Journal, 20, E1-E9.

18. Silva, J., Chambers, I., Pollard, S., \& Smith, A. (2006). Nanog promotes transfer of pluripotency after cell fusion. Nature, 441, 997-1001

19. Ivanova, N., Dobrin, R., Lu, R., Kotenko, I., Levorse, J., DeCoste, C., et al. (2006). Dissecting self-renewal in stem cells with RNA interference. Nature, 442, 533-538.

20. Takahashi, K., \& Yamanaka, S. (2006). Induction of pluripotent stem cells from mouse embryonic and adult fibroblast cultures by defined factors. Cell, 126, 1-14.

21. Check, E. (2007). Dolly: A hard act to follow. Nature, 445, 802.

22. Hamatani, T., Carter, M., Sharov, A., \& Ko, M. (2004). Dynamics of global gene expression changes during mouse preimplantation development. Developmental Cell, 6, 117-131.
23. Deb, K., Sivaguru, M., Yong, H., \& Roberts, R. (2006). Cdx2 gene expression and trophectoderm lineage specification in mouse embryos. Science, 311, 992-996.

24. Zernicka-Goetz, M. (2006). The first cell-fate decisions in the mouse embryo: Destiny is a matter of both chance and choice. Current Opinion in Genetics \& Development, 16, 406-412.

25. Piotrowska, K., Wianny, F., Pedersen, R., \& Zernicka-Goetz, M. (2001). Blastomeres arising from the first cleavage division have distinguishable fates in normal mouse development. Development, 19, 3739-3748.

26. Motosugi, N., Bauer, T., Polanski, Z., Solter, D., \& Hiiragi, T. (2005). Polarity of the mouse embryo is established at blastocyst and is not prepatterned. Genes \& Development, 19, 1081-1092.

27. Kennedy, D. (2006). Editorial expression of concern. Science, $314,592$.

28. Vogel, G. (2006). Fraud investigation clouds paper on early cell fate. Science, 314, 1367-1368.

29. Wei, C., Miura, T., Robson, P., Lim, S. K., Xu, X. Q., Lee, M. Y., et al. (2005). Transcriptome profiling of human and murine ESCs identifies divergent paths required to maintain the stem cell state. Stem Cells, 23, 166-185.

30. Solter, D. (2005). Politically correct human embryonic stem cells? New England Journal of Medicine, 353, 2321-2323. 